\title{
THE
}

UNIVERSITY

University of Rhode Island

OF RHODE ISLAND

DigitalCommons@URI

1989

\section{Cumulant Methods and Short Time Propagators}

Rob D. Coalson

David L. Freeman

University of Rhode Island, dfreeman@uri.edu

Jimmie D. Doll

Follow this and additional works at: https://digitalcommons.uri.edu/chm_facpubs

Terms of Use

All rights reserved under copyright.

\section{Citation/Publisher Attribution}

Coalson, R. D., Freeman, D. L., \& Doll, J. D. (1989). Cumulant Methods and Short Time Propagators. Journal of Chemical Physics, 91(7), 4242-4248. doi: 10.1063/1.456803

Available at: http://dx.doi.org/10.1063/1.456803

This Article is brought to you for free and open access by the Chemistry at DigitalCommons@URI. It has been accepted for inclusion in Chemistry Faculty Publications by an authorized administrator of DigitalCommons@URI. For more information, please contact digitalcommons-group@uri.edu. 


\title{
Cumulant methods and short time propagators
}

\author{
Rob D. Coalson \\ Department of Chemistry, University of Pittsburgh, Pittsburgh, Pennsylvania 15260 \\ David L. Freeman \\ Department of Chemistry, University of Rhode Island, Kingston, Rhode Island 02881 \\ J. D. Dolla) \\ Los Alamos National Laboratory, Los Alamos, New Mexico 87545
}

(Received 9 May 1989; accepted 22 June 1989)

\begin{abstract}
The present paper clarifies a number of issues concerning the general problem of constructing improved short time quantum mechanical propagators. Cumulant methods are shown to be a particularly convenient tool for this task. Numerical results comparing methods based on partial averaging and on gradient approaches are presented for simple model problems and for many particle quantum fluids.
\end{abstract}

\section{INTRODUCTION}

Numerical path integral methods have been shown to be useful tools in the analysis of many-body problems. Numerous applications to problems in physics and chemistry have been reviewed elsewhere. ${ }^{1,2}$ In typical path integral formulations, the quantum mechanical character of the problem is expressed by the introduction of "auxiliary" degrees of freedom. As emphasized by Chandler and Wolynes, ${ }^{3}$ the use of these auxiliary degrees of freedom, in effect, turns the quantum-mechanical problem into an analogous classical problem in an enlarged space. The number of auxiliary degrees of freedom required for any given application is dictated by the magnitude of the quantum-mechanical effects in the problem and by the particulars of the path integral formulation used. As a practical matter, it is generally useful to minimize the number of auxiliary variables. Efforts to minimize the number of path integral degrees of freedom have centered historically around improving the quality of the "short time" propagators used. ${ }^{4-7}$ As with the related numerical problem of propagating differential equations, the efficiency of the overall approach is a tradeoff between "gains" from the use of a "higher-order" algorithm vs "losses" from the increase in numerical complexity.

In previous papers, ${ }^{8,9}$ we have discussed an advance in the Fourier path integral approach to Feynman path integral computations of quantum mechanical properties. Progress in that work stemmed from the introduction of a device termed "partial averaging" which enabled us to sum approximately the contributions of higher-order Fourier fluctuations to the quantum-mechanical paths. In fact, as more Fourier components are explicitly retained in the path description (i.e., as fewer are "partially averaged" away), the partial averaging-Fourier path integral (PA-FPI) prescription tends towards the canonical FPI prescription (i.e., the original version, as described by Feynman and $\mathrm{Hibbs}^{10}$ ). The latter is, of course, exact in the limit that the Feynman paths are parametrized by an infinite number of Fourier coefficients. The real issue then concerns the rate of convergence of the PA-FPI results with the number of Fourier series components explicitly retained in the path summation a) Present address: Department of Chemistry, Brown University, Providence, RI 02912. procedure. In this regard, it was demonstrated ${ }^{8,9}$ by numerical examples that partial averaging consistently produced improvements, often dramatic, for problems in both quantum thermodynamics and real time dynamics.

Part of the appeal of partial averaging arises from its suggestive nature. In particular, in addition to the implementation primarily pursued in previous papers, the partial averaging formalism was shown to be an elegant method for deducing improved small argument propagators, i.e., small $\beta$ approximations to $\left\langle x_{f}|\exp (-\beta H)| x_{0}\right\rangle$, which are accurate for larger values of $\beta$. Although a number of details of partial averaging methodology have been discussed previously, it appears appropriate to consider the subject again here. Our present purpose is twofold: first we wish to reinforce some of the ideas developed previously in Ref. 9, and, second, we wish to extend these ideas in ways suggested in that work. Our presentation is structured in the following way: In Sec. II the cumulant approach to improved small argument propagators is reviewed. We examine the logarithm of the ratio of the propagator to free particle propagator and show how to construct a power series in small argument $\beta$. Terms through $O\left(\beta^{3}\right)$ are worked out explicitly, and certain results are confirmed for the simple harmonic oscillator potential. In Sec. III, the analogous treatment of the general multidimensional case is given. In Sec. IV, we show that the improved small argument propagator which is accurate through order $n$ solves the Schrödinger equation to that order, in the sense appealed to by Feynman and Hibbs. ${ }^{10}$ Indeed, some simplifications arise in the propagator formulas when the criteria of Feynman and Hibbs is used to affix the accuracy of the approximation. Finally, Sec. V presents some illustrative numerical examples.

\section{CUMULANT APPROACH TO IMPROVED SMALL ARGUMENT PROPAGATORS IN ONE DIMENSION}

In the present section, we will review the cumulant approach for the calculation of small argument propagators. For simplicity we will initially restrict attention to one-dimensional applications. The generalization to many dimensions is straightforward and is considered in Sec. III.

We seek to compute the quantum-mechanical propagator in imaginary time $\rho_{\beta}\left(x_{f}, x_{0}\right)=\left\langle x_{f}|\exp (-\beta H)| x_{0}\right\rangle$ as- 
sociated with the Hamiltonian operator $H=p^{2}$ / $2 m+V(x)$. According to the Fourier path integral prescription, ${ }^{10,11}$

$$
\begin{aligned}
& \frac{\rho_{\beta}\left(x_{f}, x_{0}\right)}{\rho_{\beta}^{\mathrm{fp}}\left(x_{f}, x_{0}\right)} \\
& =\frac{\int_{-\infty}^{\infty} d \mathbf{a} \exp \left(-\sum_{k=1}^{\infty} a_{k}^{2} / 2 \sigma_{k}^{2}-\beta \int_{0}^{1} d u V(q(u))\right)}{\int_{-\infty}^{\infty} d \mathbf{a} \exp \left(-\sum_{k=1}^{\infty} a_{k}^{2} / 2 \sigma_{k}^{2}\right)} .
\end{aligned}
$$

Here $\rho_{\beta}^{\mathrm{fp}}$ is the free partial propagator

$\rho_{\beta}^{\mathrm{fp}}\left(x_{f}, x_{0}\right)=\left[\frac{m}{2 \pi \hbar^{2} \beta}\right]^{1 / 2} \exp \left\{-m\left(x_{f}-x_{0}\right)^{2} / 2 \hbar^{2} \beta\right\}$,

$q(u)$ is the Fourier path

$$
q(u)=x_{0}+\left(x_{f}-x_{0}\right) u+\sum_{k=1}^{\infty} a_{k} \sin (k \pi u),
$$

and $\sigma_{k}^{2}=2 \beta \hbar^{2} / m \pi^{2} k^{2}$ determines typical fluctuations in the $k$ th Fourier component of free particle paths at a particular temperature. If an infinite number of Fourier coefficients are used to represent the paths $q(u)$, then the prescription is formally exact. Equation (2.1) is of the form of an average of an exponential over a probability distribution in the Fourier path variables $\left\{a_{k}\right\}$. Schematically,

$$
\frac{\rho_{\beta}\left(x_{f}, x_{0}\right)}{\rho_{\beta}^{\mathrm{fp}}\left(x_{f}, x_{0}\right)}=\left\langle\exp \left(-\beta \int_{0}^{1} d u V(q(u))\right)\right\rangle_{a},
$$

where the a averages are implied by the bracket notation.

The natural cumulant structure ${ }^{12}$ of $\mathrm{Eq}$. (2.4) is the starting point for a variety of related lines of development. One option, partial averaging, is to retain explicitly a relatively small number of low-order Fourier coefficients in the averaging process and to perform these averages with Monte Carlo methods. Averages over the high-order terms are then approximated, typically, but not necessarily, by means of first-order cumulant techniques. In this approach, improved levels of accuracy are achieved by increasing the number of Fourier terms treated explicitly while utilizing a low-order cumulant expansion. Another option, described in greater detail below, is to utilize the cumulant structure of Eq. (2.4) to eliminate explicit consideration of all Fourier terms. In this approach, increased accuracy is achieved by extending the cumulant expansion of the right-hand side of Eq. (2.4) to higher orders. This latter approach leads directly to a $\beta$ expansion of Eq. (2.4), as we now demonstrate.

Using standard methods, ${ }^{12} \mathrm{Eq}$. (2.4) can be written as

$$
\frac{\rho_{\beta}\left(x_{f}, x_{0}\right)}{\rho_{\beta}^{\mathrm{fp}}\left(x_{f}, x_{0}\right)}=\exp \left(\sum_{m=1}^{\infty} \frac{(-\beta)^{m}}{m !} K_{m}\right),
$$

where $K_{m}$ is the $m$ th-order cumulant for the function $\int_{0}^{1} d u V(q(u))$. The connections between the cumulants and the associated power moments

$$
M_{m}=\left\langle\left(\int_{0}^{1} d u V(q(u))\right)^{m}\right\rangle_{a}
$$

are well known. Specifically, the first few cumulants are giv- en in terms of the power moments by

$$
\begin{aligned}
& K_{1}=M_{1}, \\
& K_{2}=M_{2}-M_{1}^{2}, \\
& K_{3}=M_{3}-3 M_{1} M_{2}+2 M_{1}^{3} .
\end{aligned}
$$

General expressions for arbitrary order are available. ${ }^{12}$

By inspection we see that the cumulant expression (2.5) is almost a direct $\beta$ expansion of the logarithm of the ratio of the quantum-mechanical propagator to its free particle counterpart. It would be a direct $\beta$ expansion were the cumulants themselves temperature independent. The cumulants involve $\beta$, however, since the size of the quantum-mechanical fluctuations involved are temperature dependent. Explicitly, a gradient expansion about the reference path $q_{0}(u)=x_{0}+\left(x_{f}-x_{0}\right) u$ in the expressions for the moments reveals that the first- and second-order cumulants are given by

$$
\begin{aligned}
K_{1}= & \int_{0}^{1} d u\left\{V\left(q_{0}(u)\right)+\left(\beta \hbar^{2} / 2 m\right) V^{\prime \prime}\left(q_{0}(u)\right) u(1-u)\right. \\
& \left.+\left(\beta^{2} \hbar^{4} / 8 m^{2}\right) V^{\prime \prime \prime}\left(q_{0}(u)\right) u^{2}(1-u)^{2}+O\left(\beta^{4}\right)\right\}
\end{aligned}
$$

$$
\begin{aligned}
K_{2}= & \int_{0}^{1} d u d u^{\prime}\left\{\left(\beta \hbar^{2} / m\right) u_{<}\left(1-u_{>}\right) V^{\prime}\left(q_{0}(u)\right)\right. \\
& \left.\times V^{\prime}\left(q_{0}\left(u^{\prime}\right)\right)+O\left(\beta^{2}\right)\right\} .
\end{aligned}
$$

In deriving Eqs. (2.8), we have used the fact that

$$
\sum_{k=1}^{\infty} \sigma_{k}^{2} \sin (k \pi u) \sin \left(k \pi u^{\prime}\right)=\frac{\beta \hbar^{2}}{m} u_{<}\left(1-u_{>}\right)
$$

where $u_{<}\left(u_{>}\right)$is the smaller (or larger) of $u$ and $u^{\prime}$.

That the second-order cumulant begins with contributions of order $\beta$ is a special case of a more general result. The averages over the Fourier coefficients in the cumulants involve probability distributions that become arbitrarily sharp (around $a_{k}=0$ ) as $\beta \rightarrow 0$. This sharpness in the probability distributions implies that all cumulants beyond first order thus vanish as $\beta \rightarrow 0$. From this argument, we see that the constant term in a high temperature power series expansion of the general $n$ th-order cumulant $K_{n}, n>1$, must vanish. Stated differently, the second-order cumulant cannot contribute to a $\beta$ expansion of the exponent of Eq. (2.5) through order $\beta^{2}$, the third-order cumulant through order $\beta^{3}$ etc. Retaining only the first-order cumulant term in the exponent of the right-hand side of (2.5) thus results in the exponent being computed accurately through order $\beta^{2}$. The correctness of this result, initially discussed in detail in Ref. 9 , is independent of the detailed form of the potential as long as the cumulant expansion in Eq. (2.5) has no special pathologies. This point appears to have caused confusion in some recently published work. ${ }^{13}$ Its validity for the special case of the harmonic oscillator is explicitly demonstrated below.

Using the above results, we can readily write down approximations to $\mathrm{Eq}$. (2.5) correct through various orders in $\beta$ in the exponent. Specifically, through order $\beta^{3}$, 


$$
\begin{aligned}
\frac{\rho_{\beta}\left(x_{f}, x_{0}\right)}{\rho_{\beta}^{\mathrm{fp}}\left(x_{f}, x_{0}\right)}= & \exp \left(-\beta \int_{0}^{1} d u V\left(q_{0}(u)\right)-\frac{\beta^{2} \hbar^{2}}{2 m}\right. \\
& \left.\times \int_{0}^{1} d u u(1-u) V^{\prime \prime}\left(q_{0}(u)\right)\right) \\
& -\frac{\beta^{3} \hbar^{4}}{8 m^{2}} \int_{0}^{1} d u u^{2}(1-u)^{2} V^{\prime \prime \prime}\left(q_{0}(u)\right) \\
& +\frac{\beta^{3} \hbar^{2}}{2 m} \int_{0}^{1} d u d u^{\prime} u_{<}\left(1-u_{>}\right) \\
& \times V^{\prime}\left(q_{0}(u)\right) V^{\prime}\left(q_{0}\left(u^{\prime}\right)\right) .
\end{aligned}
$$

We note the relative ease with which the results in Eq. (2.10) were obtained using the cumulant approach. We also note that these results necessarily agree exactly with the corresponding third-order results quoted by Makri and Miller. ${ }^{14}$ We again emphasize that the results through order $\beta^{2}$ in the exponent of the right-hand side of Eq. (2.10) arise entirely from the first-order cumulant term while the second-order cumulant's contribution begins at $\operatorname{order} \beta^{3}$.

Before proceeding, it is useful to consider the first-order cumulant result in greater detail. From previous work $k^{9}$ and from the above discussion, we know that truncating the cumulant expansion of Eq. (2.5) at first order correctly reproduces the exponent of the right-hand side of Eq. (2.5) through terms of order $\beta^{2}$. That is,

$$
\frac{\rho_{\beta}\left(x_{f}, x_{0}\right)}{\rho_{\beta}^{\mathrm{fp}}\left(x_{f}, x_{0}\right)}=\exp \left(-\beta K_{1}+O\left(\beta^{3}\right)\right),
$$

where $K_{1}$ is given by [cf. Eq. (2.7a)]

$$
K_{1}=\int_{0}^{1} d u\left\langle V\left(q_{0}(u)+\sum_{k=1}^{\infty} a_{k} \sin (k \pi u)\right)\right\rangle_{a}
$$

It is easy to show (cf. Refs. 8 and 9) that the infinite-dimensional Gaussian average over the $a_{k}$ variables in Eq. (2.12) can be reduced exactly to a single-dimensional Gaussian average. Specifically,

$$
K_{1}=\int_{0}^{1} d u V_{\mathrm{eff}}(u)
$$

where

$V_{\mathrm{eff}}(u)=\frac{\int_{-\infty}^{\infty} d p \exp \left(-p^{2} / 2 \sigma^{2}(u)\right) V\left(q_{0}(u)+p\right)}{\int_{-\infty}^{\infty} d p \exp \left(-p^{2} / 2 \sigma^{2}(u)\right)}$

and where

$$
\sigma^{2}(u)=\frac{\beta \hbar^{2}}{m} u(1-u)
$$

For some potentials, the Gaussian average in Eq. (2.14) can be performed analytically. In general, however, this will not be possible. Progress in this more general case can be made by noting that the use of the exact first-order cumulant result (2.11) is itself accurate only through order $\beta^{2}$. Accuracy to the same order in $\beta$ is achieved if we replace $V_{\text {eff }}$ by an approximate result, itself accurate to order $\beta$. From Eq. (2.14), we find that
$V_{\mathrm{eff}}(u)=V\left(q_{0}(u)\right)+V^{\prime \prime}\left(q_{0}(u) \mid \sigma^{2}(u) / 2+O\left(\beta^{2}\right)\right.$.

Thus we have the result that

$$
K_{1}=\bar{V}\left(x_{f}, x_{0}\right)+O\left(\beta^{2}\right)
$$

where

$$
\begin{aligned}
\bar{V}\left(x_{f}, x_{0}\right)= & \int_{-1 / 2}^{1 / 2} d w\left\{\dot{V}\left(x_{\mathrm{fp}}(w)\right)\right. \\
& \left.+\frac{\beta \hbar^{2}}{2 m}\left(\frac{1}{4}-w^{2}\right) V^{\prime \prime}\left(x_{\mathrm{fp}}(w)\right)\right\}
\end{aligned}
$$

and where

$$
x_{\mathrm{fp}}(w) \equiv\left(x_{0}+x_{f}\right) / 2+\left(x_{f}-x_{0}\right) w .
$$

Note that in Eqs. (2.18), the "time" integral has been shifted in order to make clear that the final result is symmetrical with respect to interchange of $x_{0}$ and $x_{f}$. Through order $\beta$, Eqs. (2.18a) and (2.8a) are identical.

In order to evaluate Eq. (2.18) more explicitly, the precise functional form for $V$ must be specified. It is clear that the ultimate structure of the right-hand side of Eq. (2.18) will be rather complicated in general. This raises a question concerning the utility of the "improved" small argument propagator $\quad \rho_{\beta}\left(x_{f}, x_{0}\right) \approx \rho_{b}^{\mathrm{fp}}\left(x_{f}, x_{0}\right) \exp \left[-\beta \bar{V}\left(x_{f}, x_{0}\right)\right]$. Fortunately, there is a way to extract from $\bar{V}$ in the general case an explicit and simple effective potential such that $\rho_{\beta}$ is "accurate" through $O\left(\beta^{2}\right)$. The sense in which this claim is true is somewhat different from the current sense of counting explicit powers of $\beta$ in the exponent of the right-hand side of Eq. (2.5). The modified criterion counts factors of $\left(x_{f}-x_{0}\right)$ as effectively $O\left(\beta^{1 / 2}\right)$. There is a good reason for this. With such a counting scheme, an improved small time propagator accurate through $O\left(\beta^{n}\right)$ will satisfy the Schrödinger equation through $O\left(\beta^{n}\right)$ in the manner originally considered by Feynman to prove equivalence of his path integral formulation to the Schrödinger equation. We return to this point in Sec. IV.

To close this section, we check the result (2.18) in one verifiable case, namely the simple harmonic oscillator. For $V(x)=m \omega^{2} x^{2} / 2$, Eq. (2.18) can be explicitly evaluated to yield

$$
\begin{aligned}
\frac{\rho_{\beta}\left(x_{f}, x_{0}\right)}{\rho_{\beta}^{\mathrm{fp}}\left(x_{f}, x_{0}\right)}= & \exp \left\{-\beta\left[\frac{m \omega^{2}}{4}\left(x_{0}^{2}+x_{f}^{2}\right)+\frac{m \omega^{2}}{12}\right.\right. \\
& \left.\left.\times\left(\frac{\beta \hbar^{2}}{m}-\left(x_{f}-x_{0}\right)^{2}\right)\right]+O\left(\beta^{3}\right)\right\} .
\end{aligned}
$$

The exact propagator is well known ${ }^{15}$ :

$$
\begin{aligned}
\frac{\rho_{\beta}\left(x_{f}, x_{0}\right)}{\rho_{\beta}^{\mathrm{fp}}\left(x_{f}, x_{0}\right)}= & {\left[\frac{\beta \hbar \omega}{\sinh (\beta \hbar \omega)}\right]^{1 / 2} \exp \left\{\frac{-m \omega}{2 \hbar \sinh (\beta \hbar \omega)}\right.} \\
& \times\left[\cosh (\beta \hbar \omega)\left(x_{0}^{2}+x_{f}^{2}\right)-2 x_{0} x_{f}\right] \\
& \left.+\frac{m}{2 \beta \hbar^{2}}\left(x_{f}-x_{0}\right)^{2}\right\} .
\end{aligned}
$$

Using standard identities 
$\ln (1+y)=y-\frac{y^{2}}{2}+\cdots, \quad \frac{\sinh y}{y}=1+\frac{y^{2}}{3 !}+\frac{y^{4}}{5 !}$,

$\frac{\cosh y}{\sinh y}=\frac{1}{y}+\frac{y}{3}-\frac{y^{3}}{45}+\cdots$,

$\frac{1}{\sinh y}=\frac{1}{y}-\frac{y}{6}+\frac{7 y^{3}}{360}+\cdots$,

one finds that the expansion of the right-hand side of Eq.

(2.19) is recovered exactly.

\section{CUMULANT APPROACH TO IMPROVED SMALL ARGUMENT PROPAGATORS IN $\mathbf{N}$-d}

In this section, the development given in Sec. II for onedimensional systems is extended to $N$ degrees of freedom systems governed by Hamiltonians of the form

$$
H=\sum_{i=1}^{N} \frac{p_{i}^{2}}{2 m_{i}}+V(\mathbf{x}),
$$

where $\mathrm{x}=\left(x^{(1)} \ldots x^{(N)}\right)$ is an $N$-dimensional vector of spatial coordinates and $p_{i}$ the momentum conjugate to $x^{(i)}$. As discussed previously, ${ }^{9}$ partial averaging can easily be implemented for $N$-dimensional systems because the averages are over independent sets of Gaussian variables (one set for the Fourier fluctuations in each Cartesian coordinate). Hence one readily finds the analog of the $1 D$ result given by $\mathrm{Eq}$. (2.5) to be

$$
\frac{\rho_{\beta}\left(\mathbf{x}_{f}, \mathbf{x}_{0}\right)}{\rho_{\beta}^{\mathrm{fp}}\left(\mathbf{x}_{f}, \mathbf{x}_{0}\right)}=\exp \left(\sum_{m=1}^{\infty} \frac{(-\beta)^{m}}{m !} K_{m}\right) .
$$

Here $\rho_{\beta}^{\mathrm{fp}}$ is the $N$-dimensional free particle propagator and the cumulants now involve multidimensional averages over $N$ Gaussian variables. Specifically $K_{1}=\int_{0}^{1} d u V_{\text {eff }}(u)$, where $V_{\text {eff }}(u)$ is given by

$$
V_{\text {eff }}(u)=\frac{\int_{-\infty}^{\infty} d \mathbf{p} \exp \left(-\sum_{i=1}^{N} p_{i}^{2} / 2 \sigma_{i}^{2}(u)\right) V\left(\mathbf{q}_{0}(u)+\mathbf{p}\right)}{\int_{-\infty}^{\infty} d \mathbf{p} \exp \left(-\sum_{i=1}^{N} p_{i}^{2} / 2 \sigma_{i}^{2}(u)\right)}
$$

Following the notation adopted in the 1D case $\sigma_{i}^{2}(u)$ $=\left(\beta \hbar^{2} / m_{i}\right) u(1-u)$ and the $i$ th component of $q_{0}$ is given by $\left(q_{0}\right)^{(i)}=x_{0}^{(i)}+\left(x_{f}^{(i)}-x_{0}^{(i)}\right) u$. By expanding $V$ in a multidimensional Taylor series about the free particle path $\mathbf{q}_{0}$, it is readily confirmed that

$$
K_{1}=\bar{V}\left(\mathbf{x}_{f}, \mathbf{x}_{0}\right)+O\left(\beta^{2}\right),
$$

where

$$
\begin{aligned}
\bar{V}\left(\mathbf{x}_{f}, \mathbf{x}_{0}\right)= & \int_{-1 / 2}^{1 / 2} d w\left\{V\left(\mathbf{x}_{\mathrm{fp}}(w)\right)+\sum_{i=1}^{N} \frac{\beta \hbar^{2}}{2 m_{i}}\left(\frac{1}{4}-w^{2}\right)\right. \\
& \left.\times V_{i i}^{\prime \prime}\left(\mathbf{x}_{\mathrm{fp}}(w)\right)\right\}
\end{aligned}
$$

Here $V_{i i}^{\prime \prime}$ means second derivative with respect to $x^{(i)}$ and the $i$ th component of $\mathbf{x}_{\mathrm{fp}}(w)$ is given by

$$
\left(\mathbf{x}_{f p}\right)^{(i)}=\frac{1}{2}\left(x_{f}^{(i)}+x_{0}^{(i)}\right)+\left(x_{f}^{(i)}-x_{0}^{(i)}\right) w .
$$

As in the 1D case, further evaluation of $\bar{V}$ depends on the specifics of the potential function. However, again as in 1D, a simple effective potential which can be employed to give a propagator accurate through $O\left(\beta^{2}\right)$ in the Feynman-Hibbs sense $^{16}$ does exist in the general case. This will be discussed in the next section.

Finally, we check Eq. (3.4) explicitly in the case of the many-dimensional harmonic oscillator potential $V(x)$ $=\frac{1}{2} \mathbf{x} \cdot \mathbf{K} \cdot \mathbf{x}$, where $\mathbf{K}$ is an $N \times N$ real symmetric matrix of force constants.

Then

$$
\begin{aligned}
\bar{V}\left(\mathbf{x}_{f}, \mathbf{x}_{0}\right)= & \frac{1}{2}\left[V\left(\mathbf{x}_{0}\right)+V\left(\mathbf{x}_{f}\right)\right]+\frac{1}{12}\left[\beta \hbar^{2} \sum_{i=1}^{N} \frac{K_{i i}}{m_{i}}\right. \\
& \left.-\left(\mathbf{x}_{f}-\mathbf{x}_{0}\right) \cdot \mathbf{K} \cdot\left(\mathbf{x}_{f}-\mathbf{x}_{0}\right)\right] .
\end{aligned}
$$

This results in the approximate small argument propagator

$$
\frac{\rho_{\beta}\left(\mathbf{x}_{f}, \mathbf{x}_{0}\right)}{\rho_{\beta}^{\mathrm{fp}}\left(\mathbf{x}_{f}, \mathbf{x}_{0}\right)}=\exp \left\{-\beta \bar{V}\left(\mathbf{x}_{f}, \mathbf{x}_{0}\right)+O\left(\beta^{3}\right)\right\},
$$

which from the arguments of the current section is seen to be accurate through $O\left(\beta^{2}\right)$ as indicated. This result can be checked explicitly by transforming to normal modes (diagonalizing $\mathbf{K}$ ), writing down the exact propagator as a product of normal mode (1D harmonic oscillator) propagators, expanding through $O\left(\beta^{2}\right)$ as in the 1D case, and transforming back to displacement coordinates $x$. The result contained in Eqs. (3.4) and (3.5) is thereby recovered exactly.

\section{EXPLICIT EFFECTIVE POTENTIALS FOR SOLVING THE SCHRÖDINGER EQUATION TO $O\left(\beta^{\prime}\right)$}

The purpose of the quantum propagator is, as its name suggests, to transform an initial wave function into a final one according to the Schrödinger differential equation. In coordinate space, the necessary action of the propagator can be seen by writing

$$
\psi(x, \beta)=\exp \left\{-\beta h_{x}\right\} \psi_{0}(x)
$$

with $h_{x}$ the coordinate space representation of the time-independent Hamiltonian utilized above. Clearly $\psi(x, \beta)$ is the solution of the Schrödinger equation $-\partial \psi(x, \beta) / \partial \beta$ $=h_{x} \psi(x, \beta)$ with the value $\psi_{0}(x)$ at $\beta=0$. Thus for the coordinate space propagator $\left\langle x_{f}|\exp \{-\beta H\}| x_{0}\right\rangle$ $\equiv \rho_{\beta}\left(x_{f}, x_{0}\right)$ to propagate properly, it must have the property that for any initial wave function $\psi_{0}$ :

$\int d x_{0} \rho_{\beta}\left(x_{f}, x_{0}\right) \psi_{0}\left(x_{0}\right)=\sum_{j=0}^{\infty} \frac{\left(-\beta h_{x_{f}}\right)^{j}}{j !} \psi_{0}\left(x_{f}\right)$

Of course, the small argument propagators discussed in Secs. II and III are not exact for arbitrarily large $\beta$. A reasonable gauge of the degree of accuracy that they possess is the highest power of $\beta$ on the right-hand side of Eq. (4.2) which they reproduce correctly. Again, the criterion utilized by Feynman and $\mathrm{Hibbs}^{16}$ to show that their small argument propagator [in which $\bar{V}$ is replaced by $V\left(x_{0}\right)$, or $V\left(x_{f}\right)$, or by the symmetrized version of this, or by $\left.V\left(\left[x_{0}+x_{f}\right] / 2\right)\right]$ is accurate through first order in $\beta$ was that it reproduced the right-hand side of Eq. (4.2) through $O(\beta)$. Similar arguments can be used to extract from the integral formula for $\bar{V}$ deduced in this work a simple effective potential $\widetilde{V}$, which reproduces the right-hand side of Eq. (4.2) through $O\left(\beta^{2}\right)$. 
Let us begin with some formal observations. From the discussion of Sec. II, we know that the coordinate space propagator can be expanded exactly as

$$
\begin{aligned}
\rho_{\beta}\left(x_{f}, x_{0}\right)= & {\left[\frac{m}{2 \pi \hbar^{2} \beta}\right]^{1 / 2} \exp \left\{-\frac{m}{2 \pi \hbar^{2} \beta}\left(x_{f}-x_{0}\right)^{2}\right\} } \\
& \times \exp \left(\sum_{k=1}^{\infty} \beta^{k} f_{k}\left(x_{0}, x_{f}\right)\right),
\end{aligned}
$$

where the $f_{k}$ 's are functions of $x_{0}$ and $x_{f}$, but are independent of $\beta$. A prescription for uniquely determining all the $f_{k}$ 's was given in Sec. II and $f_{1-3}$ are explicitly specified in Eq. (2.10). Once all of these functions have been determined, the right-hand side of Eq. (4.3) can be substituted into the left-hand side of Eq. (4.2) and it will then reproduce the right-hand side of Eq. (4.2) order by order in $\beta$ as indicated.

Let us carry out this procedure in the abstract. There are three steps:

(i) Expand the second exponential on the right-hand side of Eq. (4.3) in its defining power series. Call this the "potential" power series because the $f_{k}$ depend intimately on the system potential and, in particular, vanish if the potential does.

(ii) Write the $x_{0}$ dependence in both the potential power series and the initial wave function $\psi_{0}\left(x_{0}\right)$ as $x_{0}=x_{f}$ $-\left(x_{f}-x_{0}\right)$. Expand the $x_{0}$ dependence of both factors in a Taylor series about $x_{f}$ as indicated.

(iii) Multiply these factors together. They now consist of the potential and its derivatives, and the initial wave function and its derivatives, all evaluated at $x_{f}$ and multiplied by various nonnegative powers of $\left(x_{f}-x_{0}\right)$. All integrals over $x_{0}$ are of the Gaussian moment type and can be performed. The $n$th moment integrals which are nonzero (i.e., for even $n$ ) add an additional factor of $\beta^{n / 2}$ [cf. the first exponential in Eq. (4.3) for the relevant Gaussian] to any $\beta$ dependence generated via the potential power series. (Note that since $n \geqslant 0$, performing the $x_{0}$ integration over any term in the integrand can only raise the power of $\beta$ associated with this term.)

Once steps (i)-(iii) have been performed, it is simple to collect all terms of order $\beta^{j}$. Apart from the factor of $\beta^{j}$ itself, these determine a function of $x_{f}$ which, from the right-hand side of Eq. (4.2), can be identified with $\left(-h_{x}\right)^{j} \psi_{0}\left(x_{f}\right) / j$ !

From this exercise we see immediately that the function $f_{k}$ will not contribute to the final expression on the righthand side of Eq. (4.2) until order $\beta^{k}$ (this is the lowest power of $\beta$ which can be associated with $f_{k}$ in the three step procedure just outlined). Thus, truncation of the argument of the potential exponential factor in Eq. (4.3) at order $n$, i.e., what we called the $O\left(\beta^{n}\right)$ propagator in Sec. II, must necessarily satisfy the right-hand side of Eq. (4.2) through the order $\beta^{n}$. We say that it is accurate through order $\beta^{n}$ "in the Feynman-Hibbs sense."

We now apply the formal reasoning just given to deduce $\widetilde{V}$, a ( $\beta$ dependent) effective potential such that

$$
\rho_{\beta}\left(x_{f}, x_{0}\right) \approx \rho_{\beta}^{\mathrm{fp}}\left(x_{f}, x_{0}\right) \exp \left\{-\beta \widetilde{V}\left(x_{f}, x_{0}\right)\right\}
$$

is accurate through order $\beta^{2}$ in the Feynman-Hibbs sense. We know that

$$
\rho_{\beta}\left(x_{f}, x_{0}\right) \approx \rho_{\beta}^{\mathrm{f}_{\mathrm{p}}}\left(x_{f}, x_{0}\right) \exp \left\{-\beta \bar{V}\left(x_{f}, x_{0}\right)\right\}
$$

with $\bar{V}$ given in Eq. (2.18) is accurate to $O\left(\beta^{2}\right)$ in the argument of the exponential on the right-hand side, i.e., $-\beta \bar{V}\left(x_{f}, x_{0}\right)=\beta f_{1}+\beta^{2} f_{2}$ in the notation of the current section [cf. Eq. (2.10)]. We have thus just proved that the approximate propagator utilizing $\bar{V}$ in Eq. (3.3) solves the Schrödinger equation to $O\left(\beta^{2}\right)$ in the Feynman-Hibbs sense. The problem is that $\bar{V}$ is generally unwieldy. Fortunately, the preceding arguments allow us to extract from $\bar{V}$ a simple explicit function $\widetilde{V}$ which, although not accurate to $O\left(\beta^{2}\right)$ in the sense considered in Sec. II, is accurate to $O\left(\beta^{2}\right)$ in the Feynman-Hibbs sense considered in this section.

To arrive at $\widetilde{V}$, we simply count powers of $\left(x_{f}-x_{0}\right)$ appearing in $\bar{V}$ as $O\left(\beta^{1 / 2}\right)$. We know that no terms higher than $O(\beta)$ in this power counting scheme can contribute to the potential power series expansion discussed above to $O\left(\beta^{2}\right)$ or lower. Considering the 1D case first, one finds

$\widetilde{V}\left(x_{f}, x_{0}\right)=\frac{1}{2}\left[V\left(x_{0}\right)+V\left(x_{f}\right)\right]+\frac{V^{\prime \prime}}{12}\left[\frac{\beta \hbar^{2}}{m}-\left(x_{f}-x_{0}\right)^{2}\right]$.

The argument of the second derivative function in this formula has been vaguely notated on purpose. Evaluation at $x_{0}$ or $x_{f}$ gives accuracy of $\widetilde{V}$ through $O(\beta)$ [discrepancies arise naively at $O\left(\beta^{3 / 2}\right)$ ]. If we then insert the approximate small argument propagator

$$
\rho_{\beta}\left(x_{f}, x_{0}\right) \approx \rho_{\beta}^{\mathrm{f} p}\left(x_{f}, x_{0}\right) \exp \left\{-\beta \widetilde{V}\left(x_{f}, x_{0}\right)\right\}
$$

into the left-hand side of Eq. (4.2), we find that the righthand side of Eq. (4.2) is recovered exactly through $O\left(\beta^{2}\right)$.

Finally, let us turn to the multidimensional case. Equation (3.3) simplifies to

$$
\begin{aligned}
\widetilde{V}\left(\mathbf{x}_{f}, \mathbf{x}_{0}\right)= & \frac{1}{2}\left[V\left(\mathbf{x}_{0}\right)+V\left(\mathbf{x}_{f}\right)\right]+\frac{1}{12}\left[\beta \hbar^{2} \sum_{i=1}^{N} \frac{V_{i i}^{\prime \prime}}{m_{i}}\right. \\
& \left.-\left(\mathbf{x}_{f}-\mathbf{x}_{0}\right) \cdot V^{\prime \prime} \cdot\left(\mathbf{x}_{f}-\mathbf{x}_{0}\right)\right] .
\end{aligned}
$$

In Eq. (4.7), $\mathrm{V}^{\prime \prime}$ is the $N \times N$ matrix of second derivatives. As in the 1D case, the value at which all second derivatives are to be evaluated is notated vaguely because there are many possible alternatives which produce accuracy in Eq. (4.2) through $O\left(\beta^{2}\right)$. The same considerations pointed out in the 1D case apply here. In fact, when $\rho_{\beta}\left(\mathbf{x}_{f}, \mathbf{x}_{0}\right)$ $\approx \rho_{\beta}^{\mathrm{fp}}\left(\mathbf{x}_{f}, \mathbf{x}_{0}\right) \exp \left\{-\beta \widetilde{V}\left(\mathbf{x}_{f}, \mathbf{x}_{0}\right)\right\}$ is substituted into the lefthand side of Eq. (4.2), terms on the right-hand side through $O\left(\beta^{2}\right)$ are recovered. Moreover, this exercise leads to a further simplification which could not be anticipated on grounds considered above. Namely, the cross terms in $\mathbf{V}^{\prime \prime}$ are found not to contribute to $O\left(\beta^{2}\right)$ on the right-hand side of Eq. (4.2), since the factors of $\left(x_{f}^{(i)}-x_{0}^{(i)}\right)\left(x_{f}^{(j)}-x_{0}^{(j)}\right)$ multiplied by the appropriate Gaussian functions integrate to zero for $i \neq j$. Thus we arrive at our final expression for the $O\left(\beta^{2}\right)$ effective potential appropriate to the multidimensional case, namely, 


$$
\begin{aligned}
\widetilde{V}\left(\mathbf{x}_{f}, \mathbf{x}_{0}\right)= & \frac{1}{2}\left[V\left(\mathbf{x}_{0}\right)+V\left(\mathbf{x}_{f}\right)\right] \\
& +\frac{1}{12} \sum_{i=1}^{N} V_{i i}^{\prime \prime}\left[\frac{\beta \hbar^{2}}{m_{i}}-\left(x_{f}^{(i)}-x_{0}^{(i)}\right)^{2}\right] .
\end{aligned}
$$

\section{NUMERICAL EXAMPLES}

We consider now the application of the methods discussed above to a number of problems. Our basic purpose will be to compare the rates of convergence of different physical properties computed by means of various approaches.

We first examine the simple, analytic example of the harmonic oscillator and compare the results obtained by means of partial averaging with those obtained using the third-order $\beta$ expansion. Shown in Table $I$ are the errors found for a particular density matrix element $\left(x=x^{\prime}=0\right)$ computed by means of the two methods. The Trotter results in Table I utilize short time propagators based on the thirdorder $\beta$ expansion described by Makri and Miller. ${ }^{13}$ The analogous Fourier results were obtained using the partial averaging methods (first-order cumulant) described in Sec. II and in greater detail in Sec. VII of Ref. 9. We see from Table I that partial averaging is very effective at accelerating the convergence of the final results. For a given number of path integral degrees of freedom, the error in the density matrix computed via partial averaging is a factor of 5 to 10 less than that obtained using the third-order $\beta$ expansion. The level of improvement found for the partial averaging results in Table I is consistent with that seen previously ${ }^{9}$ in a number of equilibrium and dynamical applications.

We now turn to many-body applications. The additional complexity involved in such calculations has, to a degree, limited detailed comparisons of various alternative approaches. We present in Table II results from a previously unpublished study designed to compare the quality of results obtained by Trotter methods with those obtained by partial averaging approaches for a model of liquid helium. ${ }^{17}$ In order to facilitate comparison with published results, the model and the thermodynamic states chosen were the same ones studied by Pollock and Ceperley ${ }^{18}\left(\rho \sigma^{3}=0.365 ; k_{B} T / \epsilon\right.$ $=5.0$ and 0.5 ). For these temperatures, the effects of particle exchange are not likely to be significant and were not included in either the present study or in the study reported

TABLE I. Listed below are the percentage errors [100 $\times$ (computed-exact)/exact] for the ( $\left.x=0, x^{\prime}=0\right)$ harmonic oscillator density matrix element computed by means of the third-order $\beta$-expansion methods [Eq. (23) of Ref. 13] (MM) and by partial averaging (PA) methods (Ref. 9). Results are shown as a function of the number of path integral degrees of freedom $(N)$, a quantity that corresponds to the number of Fourier coefficients explicitly retained in the case of the Fourier results and to the Trotter index in the case of the gradient results. Values shown are for the particular system characterized by $\beta \hbar \omega=\pi$.

\begin{tabular}{rcc}
\hline$N$ & $M M$ & $P A$ \\
\hline 2 & 4.908 & -0.468 \\
4 & 0.363 & -0.088 \\
8 & 0.056 & -0.013 \\
16 & 0.012 & -0.002 \\
\hline
\end{tabular}

TABLE II. Listed below are the average potential energies per particle for a 64-particle Lennard-Jones model of ${ }^{4} \mathrm{He}\left(\epsilon / k_{B}=10.22 \mathrm{~K}, \sigma=2.556 \AA\right)$ at two different temperatures. Energies are in units of the Lennard-Jones well depth. The reduced density in the calculation was $\rho \sigma^{3}=0.365$. Results designated by FPI (PA) were computed using Fourier path integral (partial averaging) methods. The variable $k_{\max }$ is the number of coefficients explicitly retained in the Fourier calculations. The results of Pollock and Ceperley (PC) (Ref. 18) are shown for comparison. The PC results are based on a Trotter expansion ( of order $p$ ) in conjunction with an improved short time propagator obtained from a separate pair density matrix calculation. For comparison, the corresponding classical Monte Carlo results are $\langle V\rangle=-1.797 \pm 0.008$ at $T=51.1 \mathrm{~K}$ and $\langle V\rangle=-4.296 \pm 0.017$ at $T=5.11 \mathrm{~K}$, respectively. The Fourier results typically utilized 10000 passes consisting of one attempted Monte Carlo move for each degree of freedom.

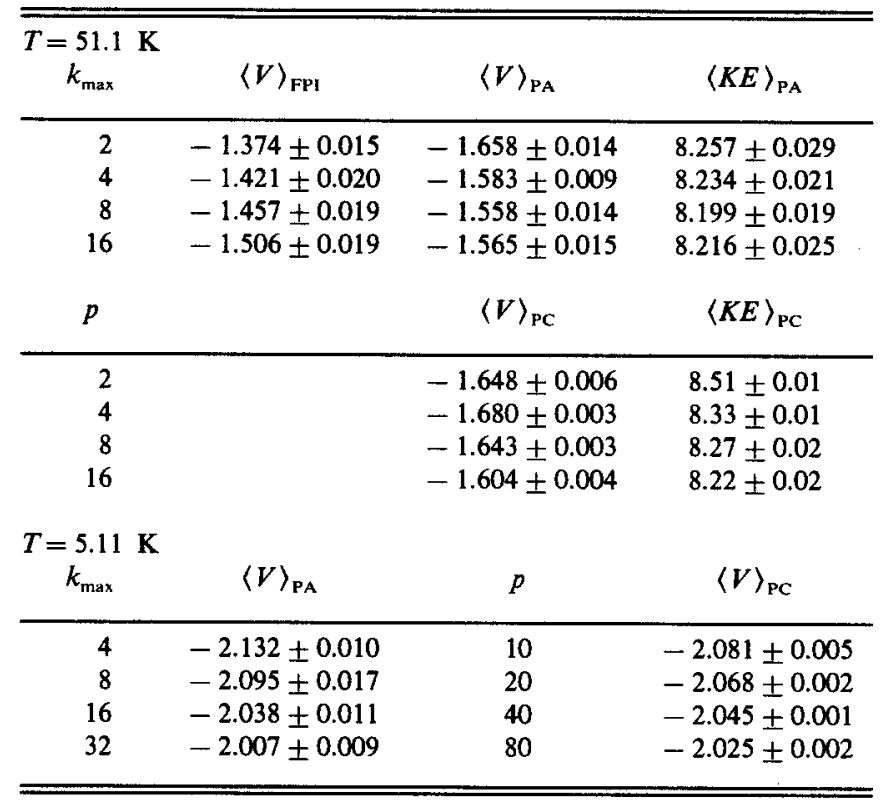

in Ref. 18. The Pollock and Ceperley results were obtained using a conventional Trotter path integral formulation with an improved short time propagator derived from a separate pair density matrix calculation. According to Pollock and Ceperley, this improved short time propagator gave "much quicker convergence" than the corresponding first-order propagator results. Although future studies with the improved short time propagator proposed in Ref. 14 will be required before a definitive conclusion can be drawn, it seems unlikely that the quality of such results would surpass that obtained using the pair density matrix approach. In fact, aspiring even to this level of improvement might be generally optimistic in light of Feynman's comments ${ }^{19}$ concerning the efficacy of including quantum-mechanical effects via such gradient methods for typical molecular interaction potentials. In Table II, we present various results obtained for a conventional 64 particle Lennard-Jones minimum image spherical cutoff model of ${ }^{4} \mathrm{He}$. The results in Table II indicate again that partial averaging is a very effective tool for accelerating the convergence of numerical path integral calculations. Other thermodynamic quantities for helium computed using the partial averaging method have been presented elsewhere. ${ }^{20,21}$ 


\section{SUMMARY}

The general area of numerical path integral methods is a rapidly changing one. Although current methods already provide an improvement tool for the analysis of many-body phenomena, new and ultimately better methods for equilibrium and dynamical applications will no doubt be discovered. The likelihood of such progress increases as we raise the level of understanding concerning the connections between various methods.

The present paper has considered a number of issues related to the task of constructing improved short time quantum-mechanical propagators. We have shown that cumulant methods are an especially effective tool for this task as well as for clarifying the connections between various alternative approaches. In this context, Fujiwara, et al. ${ }^{22}$ have presented a definitive study that considers both high temperature and Wigner-Kirkwood expansions.

We have presented numerical applications to one-dimensional as well as to many-body problems in an effort to gain insight into the relative efficiencies of various proposed methods. For the examples considered, we find the Fourier partial averaging method to be a particularly effective approach.

\section{ACKNOWLEDGMENTS}

One of us (D.L.F.) acknowledges the donors of the Petroleum Research Fund of the American Chemical Society for partial support of this work. This work was also supported in part by grants from Research Corporation and the University of Rhode Island Academic Computer Center. Also RDC acknowledges the National Science Foundation, the Petroleum Research Fund of the American Chemical So- ciety and the Alfred P. Sloan Foundation for financial support.

'For a cross section of recent activity in the area, see J. Stat. Phys. 43, 7291244 (1986).

${ }^{2}$ For a review of recent work, see B. J. Berne and D. Thirumalai, Annu. Rev. Phys. Chem. 37, 401 (1986).

${ }^{3}$ D. Chandler and P. G. Wolynes, J. Chem. Phys. 74, 4078 (1981).

${ }^{4}$ M. Suziki, Prog. Theor. Phys. 56, 1454 (1976); Commun. Math. Phys. 51, 183 (1976); 57, 193 (1977).

${ }^{5}$ H. De Raedt and B. De Raedt, Phys. Rev. A 28, 3575 (1983).

${ }^{6}$ D. Thirumalai and B. J. Berne, J. Chem. Phys. 79, 4889 (1983).

${ }^{7}$ K. S. Schweizer, R. M. Stratt, D. Chandler, and P. G. Wolynes, J. Chem. Phys. 75, 1347 (1981).

${ }^{8}$ J. D. Doll, R. D. Coalson, and D. L. Freeman, Phys. Rev. Lett. 55, 1 (1985).

${ }^{9}$ R. D. Coalson, D. L. Freeman, and J. D. Doll, J. Chem. Phys. 85, 4567 (1986).

${ }^{10}$ R. P. Feynman and A. R. Hibbs, Quantum Mechanics and Path Integrals (McGraw-Hill, New York, 1965).

${ }^{11}$ References 8 and 9. Also, D. L. Freeman and J. D. Doll, Adv. Chem. Phys. 70B, 139 (1988), and references therein.

${ }^{12}$ See, e.g., H. Risken, The Fokker-Planck Equation (Springer, Berlin, 1984), Sec. 2.2 .

${ }^{13}$ N. Makri and W. H. Miller, Chem. Phys. Lett. 151, 1 (1988).

${ }^{14}$ N. Makri and W. H. Miller, J. Chem. Phys. 90, 904 (1989).

${ }^{15}$ R. P. Feynman, Statistical Mechanics (Benjamin, Reading, MA, 1972), Sec. (2.5).

${ }^{16}$ Reference 10, Chap. 4

${ }^{17}$ Gradient partial averaging methods of Ref. 9, Sec. I were used in the present study. It should be noted that gradient partial averaging treats only those quantum mechanical effects not already explicitly included in the numerical calculation. This is in contrast to more traditional approaches where one is attempting to include all quantum-mechanical effects by means of the gradient expansion.

${ }^{18}$ E. L. Pollock and D. M. Ceperley, Phys. Rev. B 30, 2555 (1984).

${ }^{19}$ Reference 10, p. 282.

${ }^{20}$ J. D. Doll and D. L. Freeman, Science 234, 1356 (1986).

${ }^{21}$ D. L. Freeman, R. D. Coalson, and J. D. Doll, J. Stat. Phys. 43, 931 (1986).

${ }^{22}$ Y. Fujiwara, T. A. Osborn, and S. F. J. Wilk, Phys. Rev. A 25, 14 (1982). 
The Journal of Chemical Physics is copyrighted by the American Institute of Physics (AIP). Redistribution of journal material is subject to the AIP online journal license and/or AIP copyright. For more information, see http:/ojps.aip.org/jcpo/jcpcr/jsp Copyright of Journal of Chemical Physics is the property of American Institute of Physics and its content may not be copied or emailed to multiple sites or posted to a listserv without the copyright holder's express written permission. However, users may print, download, or email articles for individual use. 
The Journal of Chemical Physics is copyrighted by the American Institute of Physics (AIP). Redistribution of journal material is subject to the AIP online journal license and/or AIP copyright. For more information, see http://ojps.aip.org/jcpo/jcper/jsp 